\title{
GSP08
}

\section{Optimized Wellbore Positioning Delivers Section $100 \%$ in the Pay Zone and Reduces Operational Time by 12 Days}

\author{
P. Johnson* (Halliburton Sperry Drilling) \& F. Hveding (Halliburton)
}

\section{SUMMARY}

The InSite ADR sensor together with the StrataSteer 3D service provided a fully compensated, multipledepth resistivity measurement, real-time petrophysical evaluation and stratigraphic navigation solution in one package. A suite of LWD sensors run in conjunction with this service package enabled 24-hour realtime modelling by geosteering specialists who constantly evaluated and correlated all the information acquired to maintain the well on the right path and to help ensure the pre-established target was delivered.

Having the ability to detect approaching bed boundaries 18 feet from the tool, the InSite ADR sensor enabled the operator make adjustments in real time to stay in the target zone and allowed for increased drilling speed. Geological uncertainties were reduced and drilling operations were optimized, delivering an improvement net pay.

Sperry delivered the 1,934 meter (6,345 feet) section in only three days, 12 days ahead of schedule, with an average rate of penetration of 34.32 meters per hour. The total amount saved on this section alone was in the range of several hundred thousand USD. But the real value comes into play when you repeatedly drill wells ahead of schedule allowing more wells drilled per yearly budget, and get more wells on production earlier than estimated. 


\section{Introduction}

The hydrocarbons in the Musallim Field in Oman are trapped in the Shuaiba Formation limestone and sealed by overlying shales. An operator in this field needed to drill within the top two meters of the limestone while avoiding the shales at the top, and the high water saturation below the target zone. Many fractures, faults and channels are present that make drilling control extremely difficult in some areas. The operator wanted to improve on the 21day delivery time of a previous well, avoid any unplanned reservoir exits and be able to evaluate the geology upon completion to develop strategies for future projects.

\section{Solution}

Sperry Drilling services deployed an optimized drilling and geosteering solution that featured the Geo-Pilot ${ }^{\mathbb{B}} 5200$ series rotary steerable system, StrataSteer ${ }^{\mathbb{B}}$ 3D geosteering service, InSite ADR ${ }^{\mathrm{TM}}$ azimuthal deep resistivity sensor and suite of measurement/logging-whiledrilling $(\mathrm{M} / \mathrm{LWD})$ sensors.

The InSite ADR sensor together with the StrataSteer 3D service provided a fully compensated, multiple-depth resistivity measurement, real-time petrophysical evaluation and stratigraphical navigation solution in one package. A suite of LWD sensors run in conjunction with this service package enabled 24-hour real-time modelling by geosteering specialists who constantly evaluated and correlated all the information acquired to maintain the well on the right path and to help ensure the pre-established target was delivered.

The StrataSteer service integrates digital 3D geological earth models, directional well plans, petrophysical models and real-time LWD sensor data into a dynamic, interactive and intuitive geosteering application. It is the heart of Sperry's wellbore positioning service, crucial to placing a well, especially in smaller targets that often require complex well paths and more accurate wellbore positioning.

The InSite ADR LWD sensor provides over 2,000 unique measurements for both precise wellbore placement and more accurate petrophysical analysis. Deep reading (up to 18 feet into the formation), and directional images give early warning of approaching bed boundaries, allowing wellbore positioning in the most productive part of the reservoir. As bed dip changes along the course of a long horizontal section, the well trajectory can be corrected to run parallel with bed boundaries at a fixed distance. It also provides a traditional multifrequency compensated resistivity measurement.

Having the ability to detect approaching bed boundaries 18 feet from the tool, the InSite ADR sensor enabled the operator make adjustments in real time to stay in the target zone and allowed for increased drilling speed. Geological uncertainties were reduced and drilling operations were optimized, delivering an improvement net pay.

The Geo-Pilot rotary steerable system drilled a longer section in a minimum number of days compared to the previous results where conventional drilling assemblies had been used. Using point-the-bit technology, the Geo-Pilot system precisely steers the wellbore in any desired direction while rotating the drill string to increase ROP and reduce drilling days. The Geo-Pilot ${ }^{\circledR}$ service delivered real-time continuous at-bit steering control and formation evaluation to provide an accurate assessment of the wellbore position at all times. 


\section{Conclusions}

Sperry delivered the 1,934 meter $(6,345$ feet) section in only three days, 12 days ahead of schedule, with an average rate of penetration of 34.32 meters per hour. The total amount saved on this section alone was in the range of several hundred thousand USD. But the real value comes into play when you repeatedly drill wells ahead of schedule allowing more wells drilled per yearly budget, and get more wells on production earlier than estimated.

The ADR sensor displayed the geology such as faults, fractures, dips and depressions in a way not possible using other tools, with the resulting lateral remaining entirely in the twometer thick pay zone. Without using the ADR sensor technology, offset wells took up to 12 days to drill the lateral section and also experienced unplanned reservoir exits. In addition to saving money from reduced drilling time, the operator can now evaluate the recorded data and optimize strategies for future projects. 